\title{
Simulating Electrochemical Performance of Solid-State Electrolyte Bilayers Characterized by FIB Tomography
}

Tanner Hamann, Jon O'Neill and Eric Wachsman

Department of Materials Science and Engineering, University of Maryland, United States

The garnet-like ceramic $\mathrm{Li}_{7} \mathrm{La}_{3} \mathrm{Zr}_{2} \mathrm{O}_{12}$ (LLZO) is a strong candidate for replacing liquid organic electrolytes due to LLZO's high ionic conductivity, good thermal stability, and non-flammable chemical structure [1]. Through surface chemistry modifications to improve LLZO-Li metal compatibility, solid-state Li-metal batteries with low area-specific resistance (ASR) became feasible [2]. Additionally, adopting a porous-denseporous multi-layer microstructure further improved LLZO battery performance by minimizing electrolyte resistance, minimizing electrolyte mass, maximizing interfacial area (thus minimizing interfacial current density), and maximizing electrode loading [3]. However, further research is necessary to understand how different microstructures affect the performance of the overall electrolyte multilayer and batteries utilizing these electrolytes. Here, we simulated the electrochemical performance of LLZObilayers with varying porosities and interface conditions and evaluated the main factors affecting bilayer operation.

LLZO porous electrolytes fabricated with varying porosities were characterized by FIB Tomography (Tescan XEIA3 with Xe source, Tescan GAIA3 with Ga source) and 3D reconstructions were created from the resulting image sets, revealing the microstructures ranged from $25.7 \%$ to $55.9 \%$ porosity [4]. The reconstructions were imported into MATLAB and cropped to a cross-section of 50 x $50 \mu \mathrm{m}$ and a thickness of $20 \mu \mathrm{m}$. Digital bilayers were constructed by adding a $5 \mu \mathrm{m}$ thick dense layer to the porous reconstructions, which were now the porous layers of the bilayers. To create digital symmetric cells, the pores in the porous layer were filled by positive metal electrode and a layer of negative metal electrode was added to the free side of the dense layer. LLZO voxels were assigned a uniform ionic conductivity, and the LLZO-electrode interface was assigned an interfacial area-specific resistance (IASR) that varied between simulations. Insulating boundary conditions were applied at any LLZO voxel surface not interfacing with LLZO or electrode voxels. A fixed potential difference was applied across the bilayer and the equilibrium electric potential within the bilayer was simulated by numerically solving the Laplace equation via the Finite Difference Method (FDM). The current density distribution was then calculated and normalized to give $1 \mathrm{~mA} / \mathrm{cm}^{2}$ at the planar electrode, simulating fixed current conditions.

Figures 1a and $2 \mathrm{a}$ show reconstructions of LLZO bilayers with $25.7 \%$ and $55.9 \%$ porosity, respectively, which represented the lowest and highest porosities studied in our previous work [4]. Current density distributions in these microstructures were calculated for 1,10 , and $100 \mathrm{ohm}-\mathrm{cm}^{2}$ IASR at the LLZO-electrode interface. For low porosity (Figure 1b, c, d), the current density was initially concentrated near the dense layer-electrode interface but spread across the porous layer-electrode interface as IASR was increased, significantly reducing interfacial current densities. For high porosity (Figure 2b, c, d), the current density distribution followed a similar pattern, but was consistently shifted closer to the dense layer-electrode interface due to the higher dense layer-electrode interfacial area and the lower effective conductivity of the more porous LLZO. Moreover, more widespread utilization of the porous layer-electrode interface also increased the total current in the porous layer and led to hot spots of high current density. The hotspots occurred at bottlenecks within the bilayer microstructure, either where LLZO particle cross-sectional area reached a local minimum or where multiple porous LLZO pathways converged. Recent reports of LLZO having high enough electronic conductivity to facilitate internal Li-metal plating suggested these hot spots would be the most probable locations for dendrite nucleation [5]. Furthermore, histogram analysis showed both low and high porosity bilayers had voxels with current densities above the global current density of $1 \mathrm{~mA} / \mathrm{cm}^{2}$ for all IASR values, while the LLZO-electrode interface only had interfacial current densities below $1 \mathrm{~mA} / \mathrm{cm}^{2}$. As IASR increased and total current in the porous layer increased, more porous layer voxels had current densities amplified above $1 \mathrm{~mA} / \mathrm{cm}^{2}$, increasing 
the risk of dendrite nucleation. These simulations assumed the bilayer had a conformal and fully active LLZOelectrode interface. Realistically, if part of the LLZO-electrode interface was rendered inert (e.g., after partial stripping of the Li-metal anodes, or when using oxide cathodes composed of separate particles), then interfacial current densities would be significantly higher. Ultimately, these hot spots limit the maximum current for safely cycling the bilayer, so choosing appropriate LLZO microstructures and establishing low ASR interfaces are key to preventing dendrite nucleation and allowing higher power densities. Using the fixed potential difference and the simulated total bilayer current, the bilayer area-specific resistances (ASR) were also calculated and found to depend primarily on the interfacial ASR between the LLZO dense layer and the planar negative electrode. In contrast, the interfacial ASR between the LLZO porous layer and the positive electrode was significantly reduced due to the higher interfacial area available for charge transfer. As a result, bilayer ASR only minorly depended on porous layer porosity.

This work demonstrated that minimizing electrolyte-electrode IASR was key to improving the performance of a solid-state electrolyte bilayer, thereby minimizing bilayer ASR and reducing current density hot spots within the electrolyte. The effects of bilayer porosity were more nuanced, only minorly affecting bilayer ASR but requiring a balance between decreasing porosity to maximize particle size and further reduce current density hot spots versus increasing porosity to maximize electrode loading and minimize electrolyte mass. [6]

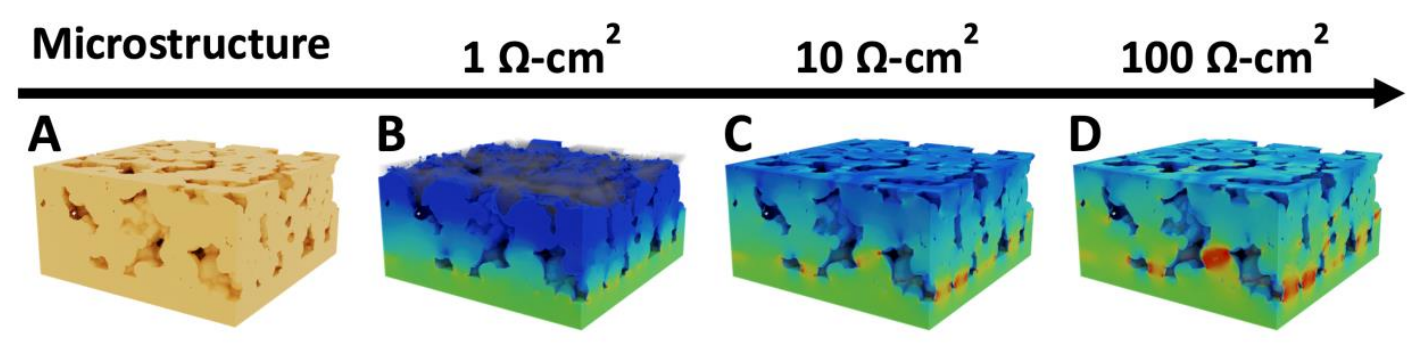

Figure 1. Figure 1: A) microstructure of LLZO bilayer with $25.7 \%$ porosity porous layer. Current density simulations with LLZO-electrode interfacial area-specific resistances of B) $1 \mathrm{ohm}-\mathrm{cm} 2$, C) $10 \mathrm{ohm}-\mathrm{cm} 2$, and D) $100 \mathrm{ohm}-\mathrm{cm} 2$. Grey represents $0 \mathrm{~mA} / \mathrm{cm} 2$, blue represents the $0.005 \mathrm{~mA} / \mathrm{cm} 2$ lower cutoff, and red represents the $2.0 \mathrm{~mA} / \mathrm{cm} 2$ upper cutoff.

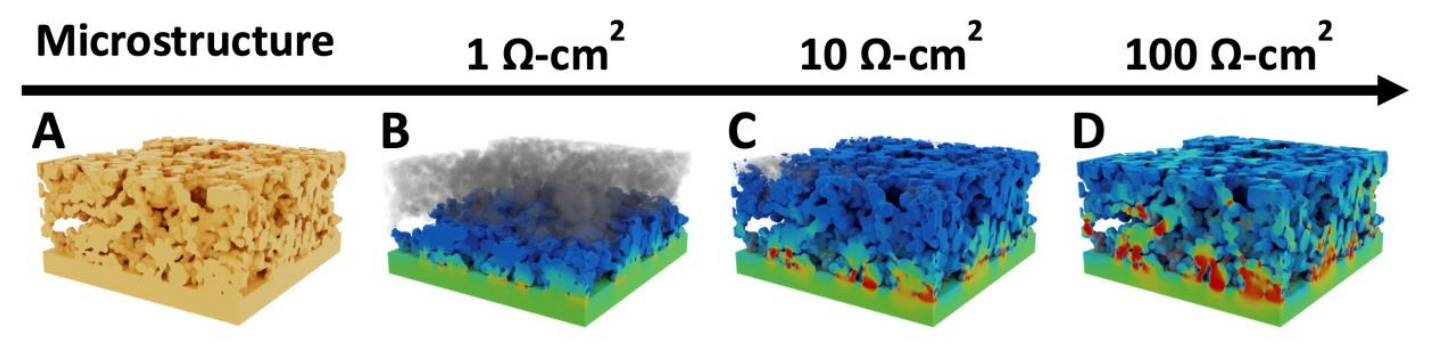

Figure 2. Figure 2: A) microstructure of LLZO bilayer with $55.9 \%$ porosity porous layer. Current density simulations with LLZO-electrode interfacial area-specific resistances of B) $1 \mathrm{ohm}-\mathrm{cm} 2$, C) $10 \mathrm{ohm}-\mathrm{cm} 2$, and D) $100 \mathrm{ohm}-\mathrm{cm} 2$. Grey represents $0 \mathrm{~mA} / \mathrm{cm} 2$, blue represents the $0.005 \mathrm{~mA} / \mathrm{cm} 2$ lower cutoff, and red represents the $2.0 \mathrm{~mA} / \mathrm{cm} 2$ upper cutoff.

\section{References}

[1] Murugan, R., et al, Angewandte Chemie (International ed. in English) 46 (2007), 7778-7781.

[2] Han, X., et al, Nature Materials 1 (2016), 572-579.

[3] Hitz, G. T., et al, Materials Today 22 (2019), 50-57.

[4] Hamann, T., et al, Advanced Functional Materials 30 (2020), 1910362. 
[5] Han, F., et al, Nature Energy 4 (2019), 187-196.

[6] The authors acknowledge funding from the Department of Energy ARPA-E, and the support of the Maryland NanoCenter and its AIMLab at UMD. 